\title{
Clinical Studies and Medical Case Reports
}

\section{Bilateral Serous Retinal Detachment as a Presenting Sign of Acute Lymphoblastic Leukemia in an Adult}

\section{Michelle C Liang* and Caroline Baumal}

New England Eye Center, Tufts Medical Center, Boston, Massachusetts, USA

\begin{abstract}
Purpose: To report a case of bilateral serous retinal detachment as a presenting sign of Acute Lymphoblastic Leukemia (ALL) in a 33-year-old man.

Methods: Case report. Main outcome measures include ocular examination, Spectral Domain-Optical Coherence Tomography (SD-OCT), color fundus photography, fluorescein angiography, and indocyanine green angiography.

Results: A 33-year-old Asian man presented with acute visual loss to $20 / 200$ in both eyes after having a fever for several days. He had no prior systemic or ophthalmological medical history. Anterior segment examination was normal in both eyes and fundus examination revealed bilateral serous retinal detachments, intraretinal hemorrhage, and cotton wool spots. Systemic work up revealed a diagnosis of Philadelphia chromosome-positive ALL.

Conclusion: Serous retinal detachment is an uncommon presenting feature among leukemia's. Although ALL is most common in childhood, with a peak incidence at 2-5 years of age, it can present in other age groups as well. Sudden appearance of unilateral or bilateral serous retinal detachment, even in an adult, warrants a thorough systemic evaluation for underlying leukemia. Prompt recognition of the disease can lead to early systemic treatment and restoration of visual function.
\end{abstract}

Keywords: Acute lymphoblastic leukemia; Philadelphia chromosome; Serous retinal detachment

\section{Introduction}

While up to $90 \%$ of people with leukemia develop retinal changes during the course of their disease, ocular findings are rare as the initial manifestation [1]. Typical ocular findings in leukemia include white-centered retinal hemorrhages, cotton-wool spots, retinal vascular sheathing, and nodular infiltrates. Serous Retinal Detachment (SRD), however, is uncommon [2].

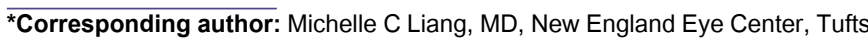
Medical Center, 800 Washington St Box 450, Boston, Massachusetts 02111 USA, Tel: 6176364600; Fax:6176364866; E-mail: mliang@tuftsmedicalcenter.org

Citation: Liang MC, Baumal C (2015) Bilateral Serous Retinal Detachment as a Presenting Sign of Acute Lymphoblastic Leukemia in an Adult. J Clin Stud Med Case Rep 2: 007.

Received: January 14, 2015; Accepted: February 21, 2015; Published: March 09, 2015
This report describes a patient who presented with bilateral SRD and systemic work up revealed a diagnosis of Philadelphia chromosome-positive $(\mathrm{Ph}+)$ Acute Lymphoblastic Leukemia (ALL).

\section{Case Report}

A 33-year-old Asian man with no pertinent medical history presented with sudden onset bilateral blurred vision, mild headaches, and low-grade fever for two days. His vision was 20/200 in both eyes. There was no evidence of intraocular inflammation. Fundus examination revealed bilateral serous retinal detachments involving the fovea (Figure 1A \& B) with scattered intraretinal hemorrhages and cotton wool spots. Fluorescein angiography showed multifocal hyper fluorescent spots centered around the optic nerve and in the nasal and superior macula in both eyes (Figure 1C \& D). Optical coherence tomography disclosed a large amount of subretinal fluid bilaterally (Figure 1E \& F). Indocyanine green angiography was unremarkable.

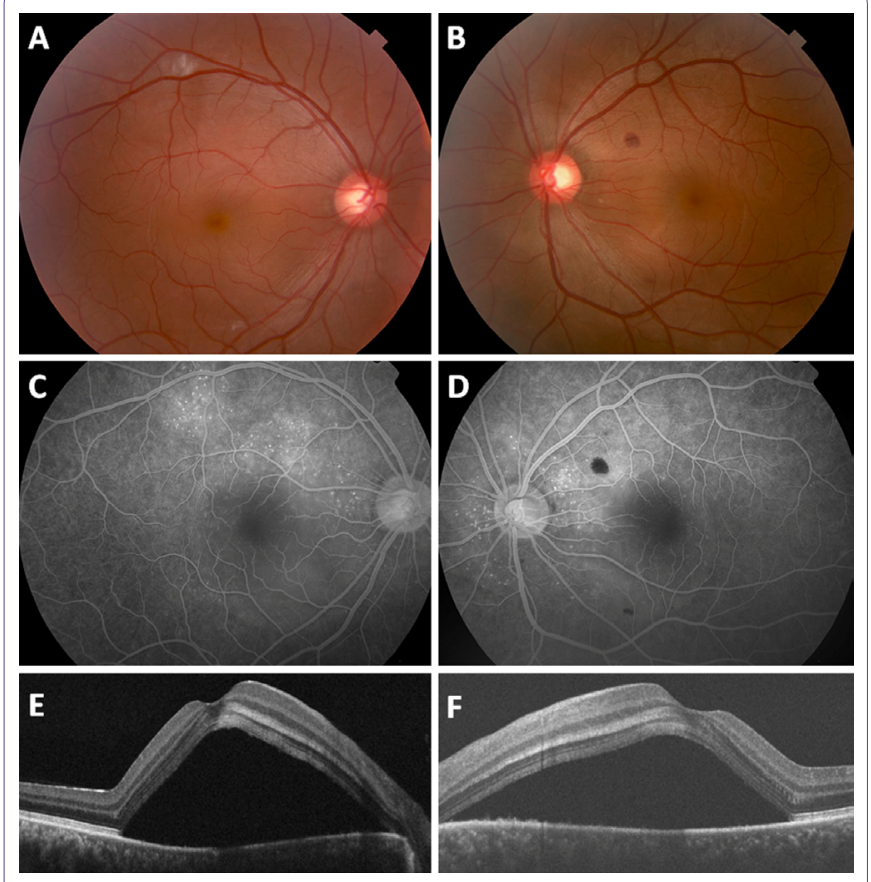

Figure 1(A-F): Presentation $(A, B)$ fundus photography showed a serous macular detachment in each eye with intraretinal hemorrhages and cotton wool spots. (C,D) Fluorescein angiography demonstrated multifocal spots of hyper fluorescence around the nerve and macula bilaterally. $(E, F)$ Cirrus SD-OCT revealed diffuse sub retinal fluid in both eyes.

Systemic work up revealed a white blood cell count of $50.8 \mathrm{~K} / \mu \mathrm{L}$ with $87 \%$ lymphoblast's, hemoglobin of $13.9 \mathrm{G} / \mathrm{DL}$, and platelets of $7 \mathrm{~K} / \mu \mathrm{L}$. Peripheral blood flow cytometry showed $74 \%$ blasts positive for CD34, HLA-DR, CD10, and CD19, and negative for myeloid markers CD33 and CD13. Coagulation screening tests were unremarkable. Bone marrow biopsy revealed $>95 \%$ blasts and cytogenetic and FISH analysis showed at $(9 ; 22)$ confirming the diagnosis of Ph+ - ALL. Imaging of the head did not detect a tumor or hemorrhage. After induction with chemotherapy, there was rapid resolution of the subretinal fluid and his vision returned to 
20/20 bilaterally with resolution of all ocular symptoms. He unfortunately suffered a relapse of disease ten months after presentation. Interestingly, he presented with unilateral blurred vision to $20 / 50$ in the left eye and was found to have a unilateral SRD (Figure 2). Despite additional chemotherapy, he developed progressive disease and has since entered hospice care.
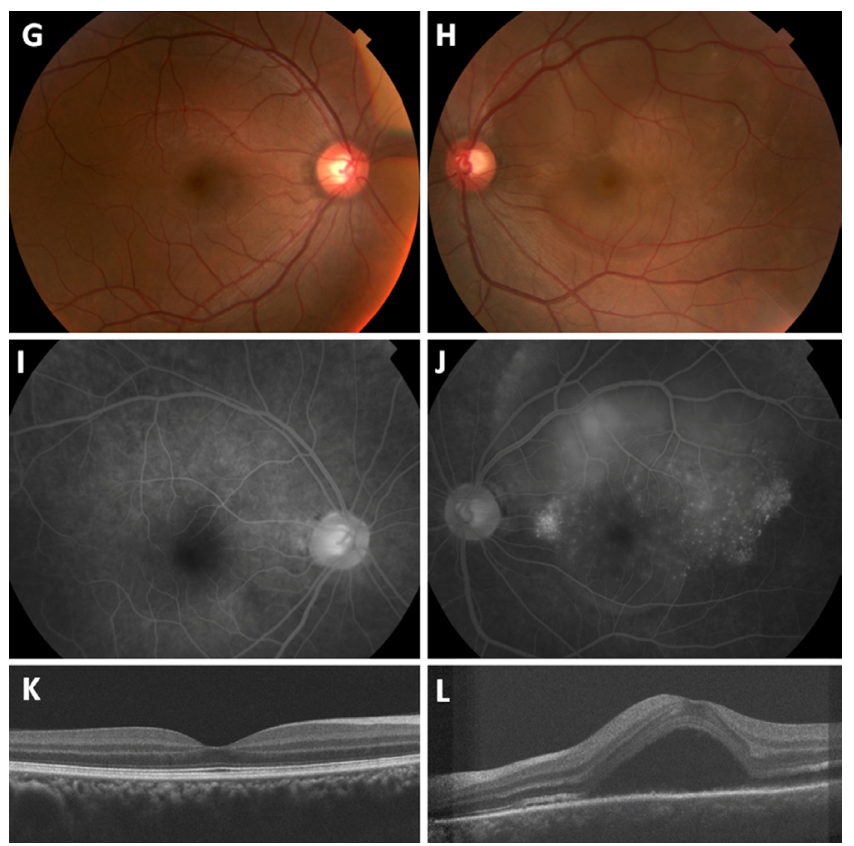

Figure 2: Recurrence $(A, B)$ Fundus imaging demonstrated a serous retinal detachment over the superior arcade and macula in the left eye. The right eye was unremarkable. (C,D) Multiple punctate areas of hyper fluorescence were seen in the macula on fluorescein angiography in the left eye only. $(E, F)$ Cirrus SD-OCT showed no abnormalities in the right eye and a recurrence of sub retinal fluid in the left eye.

\section{Discussion}

Leukemias are systemic hematologic disorders that potentially may affect all organs of the body. Some of the posterior manifestations of leukemia in the eye could be attributed to the associated hematological abnormalities such as anemia, thrombocytopenia, hyper viscosity states, or opportunistic infections [1]. Ocular findings include intraretinal hemorrhages, cotton wool spots, white-centered hemorrhages, leukemic infiltrates, central retinal vein occlusion, proliferative retinopathy with vitreous hemorrhage, and optic nerve head infiltration $[3,4]$.

It is uncommon, however, for ALL to present with visual complaints. Specifically, serous detachment of the macula as the initial sign of ALL has only rarely been reported [2,5-9]. All cases except for one in a 12-year-old boy were bilateral, [2] and only one was also found to be Philadelphia chromosome-positive [8]. The macular detachment in ALL has typically been reported to be shallow. The mechanism is hypothesized to be due to leukemic infiltration into the choroid producing decreased blood flow in the choriocapillaris. The resulting ischemia to the overlying RPE and disruption of the intercellular tight junctions is thought to lead to the development of an overlying serous detachment [2]. The involvement of the choroid by leukemic cells tends to be perivascular [10] and choroidal thickness has also shown to be significantly increased compared to normal [11]. SD-OCT in our case revealed diffuse SRF in the macula with the appearance of a thickened choroid. As far as the authors are aware, this is one of the few cases to demonstrate the detachment on SD-OCT imaging and the first in a patient with Ph+ ALL. We are also not aware of any other cases that presented initially with bilateral serous detachments and subsequently a unilateral serous detachment as a sign of recurrence.

The differential diagnosis of bilateral SRD includes central serous choroidopathy, Harada's syndrome, scleritis, uveal effusion, age-related macular degeneration, and metastatic neoplasm. While leukemia has been reported to masquerade as other ocular and systemic disorders, [12,13] clinical exam and fluorescein angiography can help narrow the differential. Central serous choroidopathy is high on the differential in the presence of sub retinal fluid and a thick choroid, especially in a young man. However, it is less likely to be associated with retinal hemorrhages and cotton wool spots. Fluorescein imaging in this case was similar to what one would expect with Harada's, however there was no associated inflammation or other systemic findings to support this diagnosis. The systemic work up in this patient was crucial to making the correct diagnosis. The intraocular manifestations of leukemia are usually treated with systemic chemotherapy, and ocular radiation is used for unresponsive cases.

In conclusion, serous macular detachment may be the initial sign of ALL, among other types of leukemias. A sudden appearance of unilateral or bilateral SRD, even in an adult, warrants a thorough systemic screening for underlying leukemia. Prompt recognition of the disease leads to early systemic treatment and may help restore visual function.

\section{References}

1. Kincaid MC, Green WR (1983) Ocular and orbital involvement in leukemia. Surv Ophthalmol 27: 211-232.

2. Stewart MW, Gitter KA, Cohen G (1989) Acute leukemia presenting as a unilateral exudative retinal detachment. Retina 9: 110-114.

3. Schachat AP, Markowitz JA, Guyer DR, Burke PJ, Karp JE, et al. (1989) Ophthalmic manifestations of leukemia. Arch Ophthalmol 107: 697-700.

4. Camera A, Piccirillo G, Cennamo G, Tranfa F, Rosa N, et al. (1993) Optic nerve involvement in acute lymphoblastic leukemia. Leuk Lymphoma 11: 153-155.

5. Fackler TK, Bearelly S, Odom T, Fekrat S, Cooney MJ (2006) Acute lymphoblastic leukemia presenting as bilateral serous macular detachments. Retina 26: 710-712.

6. Golan S, Goldstein M (2011) Acute lymphocytic leukemia relapsing as bilateral serous retinal detachment: a case report. Eye (Lond) 25: 1375-1378.

7. Vangheluwe O, Ducasse A, Culioli B, Kwong FH, Segal A (1990) [Macular manifestations of acute lymphoblastic leukemia. A case report]. Bull Soc Ophtalmol Fr 90: 677-682.

8. Kim J, Chang W, Sagong M (2010) Bilateral serous retinal detachment as a presenting sign of acute lymphoblastic leukemia. Korean $\mathrm{J}$ Ophthalmol 24 : 245-248.

9. Chinta S, Rani PK, Manusani U (2012) Bilateral exudative retinal detachment as a presenting sign of acute lymphoblastic leukemia. Middle East Afr J Ophthalmol 19: 410-412.

10. ALLEN RA, STRAATSMA BR (1961) Ocular involvement in leukemia and allied disorders. Arch Ophthalmol 66: 490-508.

11. Caillaux V, Querques G, Ramahefasolo C, Darugar A, Souied EH (2013) Bilateral macular serous retinal detachment revealing acute myeloblastic leukemia. Retin Cases Brief Rep 7: 62-66.

12. Yang HK, Yu HG (2009) Acute lymphoblastic leukemia manifesting as acute Vogt-Koyanagi-Harada disease. Korean J Ophthalmol 23: 325-328.

13. AlZamil WM (2014) Lymphocytic leukemia presenting as acute Vogt-Koyanagi-Harada disease. Saudi J Ophthalmol 28: 319-321. 